\title{
Structural Challenges and Inequities in Operating Urban Indigenous Early Learning and Child Care Programs in British Columbia
}

\author{
Alison Gerlach, Shemine Gulamhusein, Leslie Varley, and Magnolia Perron
}

\begin{abstract}
Alison Gerlach is a first-generation white settler of English and Welsh descent who is privileged to have lived and worked on the unceded traditional territory of the Coast Salish peoples for the past 30 years, including the lands of the Skwxwú7mesh (Squamish) Nation where she lives with her family and the lands of the Lkwungen, Songhees, Esquimalt, and WSÁNEĆ peoples where she currently works at the University of Victoria in the School of Child \& Youth Care. Email: alisongerlach@uvic.ca
\end{abstract}

Shemine Gulamhusein is an assistant professor in the Department of Child and Youth Care at MacEwan University. Her research focuses on the beautifully complex intersections of a person's identity and how young people negotiate and navigate living in the in-between.

Leslie Varley is the executive director of the BC Association of Aboriginal Friendship Centres. Leslie is a member of the Killer Whale Clan of the Nisgaa Nation and a lifelong social justice advocate.

Magnolia Perron is from Tyendinaga Mohawk Territory and is a proud member of the Mohawks of the Bay of Quinte. She holds a graduate diploma in Indigenous policy and administration from Carleton University and a master's in Indigenous Nationhood at the University of Victoria.

Funding for urban, not-for-profit Indigenous early learning and childcare (ELCC) programs has not kept pace with a rapidly growing urban Indigenous population, increasing operational costs, and the rights of Indigenous children. In British Columbia (BC), closure of a prominent Indigenous ELCC program prompted a study of some of the key factors influencing the operation of Indigenous ELCC programs in BC. This qualitative research highlights the priorities, concerns, and recommendations for supporting the operational success of urban, not-for-profit Indigenous ELCC programs and upholding the rights of Indigenous children. These findings have relevance for Indigenous ELCC programs that are facing operational challenges in $B C$ and other jurisdictions in Canada.

Key words: early childhood; Aboriginal children; equity; Truth and Reconciliation; Indigenous governance; selfdetermination; children's rights; Aboriginal Head Start
In settler colonial societies such as Canada, quality in Indigenous ${ }^{1}$ early learning and child care (ELCC) contexts means that programs are grounded in Indigenous philosophies on raising healthy children. Indigenous knowledges are highly diverse but often share a worldview in which children's health and well-being is viewed holistically, encompassing interrelated spiritual, physical, intellectual, and social/ emotional dimensions, and extended family and community members are valued in children's care and socialization (Halseth \& Greenwood, 2019). Greenwood (2005) describes the foundations of Indigeneity as being

comprised, in part, of values that privilege interrelationships among the spiritual, the natural and the self; reflect a sacred orientation to place and space; encompass a fluidity of knowledge exchanged between past, present and future, thereby allowing for constant and dynamic knowledge growth and change; and honour language and orality as an important means of knowledge of transmission. (p. 554)

A growing number of Indigenous families are raising their children in urban centres in Canada (Statistics Canada, 2017). In British Columbia (BC), where this study took place, approximately $80 \%$ of Indigenous peoples from First 
Nation, Métis, and Inuit backgrounds are living in urban and off-reserve areas of the province (Government of British Columbia, n.d.). Indigenous people's "migration" to urban centres is frequently conveyed in public discourse as a decision to leave rural communities and cultures and assimilate into mainstream society (Newhouse \& Peters, 2003). This discourse disregards the fact that many Canadian cities are developed on long-established gathering places or settlement areas used by Indigenous peoples prior to the government forcibly displacing and relocating entire communities to reserves far from urban centres that have since been renamed (Newhouse \& Peters, 2003). In 1996, the Royal Commission on Aboriginal Peoples highlighted that many Indigenous peoples want to have healthy family lives in cities, maintain and develop distinctive cultures and traditions, and have individual and collective governance over their daily lives.

Like all children, Indigenous children's early health and development is inseparable from the well-being of their families and communities. For many Indigenous families in Canada, their well-being and everyday lives continue to be adversely impacted by colonialism. Pertinent to understanding Indigenous parental concerns about sending their children to institutional forms of ELCC (Hare \& Anderson, 2010) is the Canadian state's use of "education" as a means of assimilation and cultural genocide. For over 100 years, Indigenous children as young as 4 were forcibly removed from their homes and communities and placed in church-run residential and industrial schools (Truth and Reconciliation Commission of Canada, 2015; Wilk et al., 2017). The ongoing impacts of colonialism mean that, compared to non-Indigenous children in Canada, Indigenous children are more likely to grow up in families affected by intergenerational trauma (Reading \& Wien, 2013) and the multiple and interrelated downstream effects of poverty (Hajizadeh et al., 2018) and to be removed from their homes, cultures, and communities by the contemporary child welfare system (Tait et al., 2013).

Quality Indigenous designed and delivered ELCC programs play a vital role in providing Indigenous children with the best possible start in life (Halseth \& Greenwood, 2019). Government investment in quality Indigenous ELCC is viewed as critical to optimizing Indigenous children's health and development, redressing the attempted elimination of Indigenous peoples, cultures, and languages, and mitigating the intergenerational impacts of social and structural inequities on families and children's living conditions and life chances (Halseth \& Greenwood, 2019). The right of Indigenous children to have equitable access to Indigenous ELCC is set out in Article 30 of the United Nations Convention on the Rights of the Child (UNCRC; United Nations, 1989). As Greenwood (2016) notes:

the provisions of Article 30 are particularly relevant in nations such as Canada that are built upon a history of colonization, where for generations Indigenous children have been dispossessed of their cultures, languages, territories, family and community ties-all of the foundational elements of healthy and whole Indigenous identities. (p. 1)

However, according to a recently developed national Indigenous ELCC framework codeveloped by the Government of Canada, Assembly of First Nations, Inuit Tapiriit Kanatami, and Métis National Council (2018), urban Indigenous children and families in Canada continue to be denied equitable access to Indigenous ELCC as a result of unstable or insufficient funding, a lack of continuity in a "patchwork of programs or services" (p. 4) and a lack of Indigenous control over planning, design, and delivery of programs.

In 2018, one of the few not-for-profit Indigenous ELCC programs for Indigenous families raising young children in the greater Vancouver area of BC closed due to unsustainable funding (CTV News, 2018). This closure, and concerns about the ongoing lack of attention to and investment in urban-based Indigenous ELCC programs in this province, prompted coauthor Leslie Varley, who is the executive director (ED) of the BC Association of Aboriginal Friendship Centres (BCAAFC), to seek funding and a research partnership to explore the operational challenges 
of running quality nonprofit urban Indigenous ELCC programs in BC with the primary author, Alison Gerlach. This paper reports on some of the key findings from their community-engaged research project, highlighting the priorities, concerns, and recommendations for supporting the operational success of urban not-for-profit Indigenous ELCC programs. These findings, while focused on urban Indigenous contexts, have relevance for many Indigenous and mainstream programs that are facing operational challenges in $\mathrm{BC}$ and other jurisdictions in Canada.

\section{Urban Indigenous early learning and child care programs in Canada}

There is no lack of evidence that infants' and young children's early life experiences and relationships can have profound effects on their early brain development and health trajectory (Boyce \& Hertzman, 2018; Halfon et al., 2018; Shonkoff, 2012; UNICEF, 2014). It is also widely accepted that quality ELCC programs can support families' and children's health and well-being, mitigate the impact of early adversity on children's life course (Britto et al., 2011; Lake \& Chan, 2014), and function as important determinants of population health (Bell et al., 2013).

In the unique context of Indigenous children in a settler colonial state, "early childhood can be seen as a crucial site for reconciliation and cultural healing" (Greenwood, 2016, p. 1). When ELCC programs are anchored in local Indigenous and community cultures, languages, protocols, practices, and histories, they can play a foundational role in children's cultural identity and health trajectory and provide a pathway for the collective health and well-being of their communities and Nations (Greenwood \& de Leeuw, 2012; Greenwood \& Jones, 2015; Royal Commission on Aboriginal Peoples, 1996; Smylie \& Adomako, 2009). Protecting and enacting Indigenous children's rights to their heritage languages, cultures, and traditions in ELCC programs is in accordance with the 1989 UNCRC, the 1996 Royal Commission on Aboriginal Peoples, the 2007 United Nations Declaration on the Rights of Indigenous Peoples (UNDRIP), and the 2015 Truth and Reconciliation Commission (TRC) of Canada. Thus, Indigenous ELCC programs uphold longstanding calls and mandates by Indigenous leadership, communities, and families to reassert their roles and rights with respect to their children, which governments are obliged to uphold according to their own laws (BC Aboriginal Child Care Society, 2014a; First Nations Information Governance Centre, 2012).

In $\mathrm{BC}$ and across Canada, an increasing number of Indigenous families are accessing urban Indigenous notfor-profit ELCC programs (Government of Canada et al., 2018), including many based in Friendship Centres. Friendship Centres are caring community hubs that offer a wide range of social services and programs in a culturally safe manner for urban Indigenous families (BCAAFC, 2020). Across BC there are 25 Friendship Centres and 16 of these currently operate an ELCC program, including some that have Aboriginal Head Start in Urban and Northern Communities (AHSUNC) programs. AHSUNC is the most prominent urban-based Indigenous ELCC program in Canada, and is supported and funded at the federal level by the Public Health Agency of Canada (PHAC). However, generally speaking, regulated child care for Indigenous families living in urban centres has no special status with the federal government and has seen its funding stagnate year after year since its inception in the early 1990s (BC Aboriginal Child Care Society, 2014a).

Promising practices in Indigenous ELCC contexts include programs that are community planned, designed, and controlled, are grounded in Indigenous knowledges, cultures, and worldviews, aim to revitalize and protect language and culture and restore identity, and incorporate a holistic approach (Halseth \& Greenwood, 2019). Achieving these goals requires that programs are embedded within interdependent relational networks involving families, Elders, and community members (Greenwood \& de Leeuw, 2007). Providing Indigenous children with ELCC programs that are rooted in culture and community is a challenging process for multifaceted reasons, including the enormous diversity of Indigenous families living in urban contexts (Place, 2012) and the effects 
of colonization on the intergenerational transmission of Indigenous knowledges and cultures (Stout, 2012). Operationally, Indigenous ELCC programs are also constrained by current governance and funding structures, a lack of qualified, skilled staff (Government of Canada et al., 2018), and predetermined structural requirements that are not always inclusive of unique Indigenous attributes (Greenwood et al., 2007).

Attracting qualified early childhood educators (ECEs) is a well-established and ongoing barrier to the successful operation of quality ELCC programs in Canada (Government of Canada, 2017; Wagner et al., 2013). In the current $\mathrm{BC}$ context, ECE recruitment is a critical topic given that the provincial government's recent commitment to achieving universal childcare over the next 10 years is predicated on recruiting an additional 12,000 ECEs and other childcare professionals (Government of British Columbia, 2018a). The BC government's Early Care and Learning Recruitment and Retention Strategy (Government of British Columbia, 2018b) reinforces the need for (1) an adequate and stable workforce comprised of qualified and skilled early learning and care professionals, (2) acknowledgment of ECE as a viable, sustainable, and valued career, and (3) appropriate compensation plans and human resource strategies. As a result, there are increasing educational opportunities and ECE bursary programs in BC to incentivize and meet the projected increasing need for ECE (Government of British Columbia, 2018b). It is currently unclear how the increasing demand for urban Indigenous ELCC programs and staffing in BC is factored into the larger provincial initiatives that are currently underway in BC.

Funding for Indigenous ELCC programs has not kept pace with a rapidly growing urban Indigenous population or increasing operational costs, including infrastructure costs, a living wage with benefits for staff, and the training and resources required to support children with diverse abilities (BC Aboriginal Child Care Society, 2014a). The historical and ongoing underfunding of urban Indigenous ELCC programs speaks to unresolved jurisdictional disputes over which level of government is responsible for funding programs and services for urban Indigenous families and children (Halseth \& Greenwood, 2019). Moreover, in the context of BC, political inaction to support a transition to "the design, delivery and governance of Indigenous ELCC that is anchored in self-determination" as called for in the Indigenous ELCC Framework (Government of Canada, 2018, p. 5) disregards the United Nations Declaration on the Rights of Indigenous Peoples (UNDRIP), which was legislated by the BC government into implementation in 2019.

\section{A community-engaged research design}

Consistent with a community-engaged and participatory approach (Hacker, 2013), this research was initiated by coauthor Leslie Varley, who is a member of the Killer Whale Clan of the Nisga'a Nation in BC and, as previously mentioned, ED of the BCAAFC. The lead university research partner and primary author of this paper, Alison Gerlach, is a new settler to Canada with British ancestry. Alison has a 20-year history of collaborating with Indigenous ELCC programs and colleagues in BC as a community-engaged researcher (Gerlach, 2007; Gerlach, Browne, \& Greenwood, 2017; Gerlach \& Gignac, 2019). The two research assistants and coauthors of this paper are Shemine Gulamhusein, who is a Canadian Muslim with East African and Indian heritage who was working at the University of Victoria at the time of this study, and Magnolia Perron, who is Kanien'kehá:ka (Mohawk) and was working with the BCAAFC during this study.

The study objectives and design, including data collection methods and knowledge translation activities, were subsequently codeveloped in partnership with the researchers who came together for this project. The purpose of this exploratory qualitative study was to generate a greater understanding of how organizational and funding factors and structures shape the operation of urban Indigenous ELCC programs in BC and to provide evidenceinformed recommendations for supporting their operational success in the context of challenges with funding 
and staff recruitment and retention. Ethics approval was granted by the University of Victoria. Data collection and analysis were undertaken over a six-month period from January to June 2019, and a research summary was published in November 2019 (Gerlach et al., 2019).

\section{Recruitment}

The researchers used purposeful and snowball recruitment strategies. In January 2019, executive directors of urban Indigenous organizations that operated an ELCC program were recruited at a BCAAFC meeting and via a subsequent email invitation. Executive directors who expressed an interest in participating provided the name of the manager of their ELCC program and were informed that their manager may or may not be contacted given the scope of the study. Some executive directors and managers subsequently contacted the researchers to say that they would like to be interviewed together.

\section{Participants}

There was a total of 19 participants, including executive directors $(n=11)$, Indigenous ELCC managers ( $n=6)$, and provincial Indigenous ELCC experts $(n=2)$. Participants represented 9 distinct Indigenous ELCC programs and 4 AHSUNC programs in all five health regions of BC (Fraser Valley: $n=1$, Interior: $n=2$, Vancouver Coastal; $\mathrm{n}=2$, Northern: $\mathrm{n}=4$, and Vancouver Island: $\mathrm{n}=10$ ). The length of time these programs had been operating ranged widely; some were newly opened and others had been in operation for over 20 years, with an average of six to seven years. Participants self-identified as female $(n=15)$ or male $(n=4)$ and their experience in their current positions ranged widely, from 10 months to 24 years. Given Leslie Varley's position as ED of the BCAAFC, she was not involved in recruitment and did not have access to participants' identities or the raw data.

\section{Data collection and analysis}

Following acquisition of participants' signed consent, the researchers conducted individual or dyad semistructured interviews based on an interview guide that explored questions such as: (1) Can you tell me about some of the ways your centre has been successful in operating your child care program? (2) What are some of the operational challenges you experience, or have experienced? (3) What do you think needs to be done differently to support the successful operation of centres like yours? Six of the interviews were conducted in person including an interview with a provincial expert. The remaining interviews were conducted by phone. The researchers recorded field notes immediately following each interview. All interviews were audio-recorded, transcribed and anonymized. The researchers codeveloped a code book, and coded a sample transcript to strengthen and refine the coding process. All transcripts and field notes were subsequently coded using HyperRESEARCH ${ }^{\odot}$. The coded data were analyzed to identify interrelated themes and subthemes, which were refined in alignment with the research objectives and a further reading of the literature. All participants were given an opportunity to review and verify their transcripts and contact the researchers if they wished to add or change any details. A discussion about the preliminary findings and recommendations took place at a BCAAFC meeting that the ED participants attended. A similar discussion of the findings took place with the ELCC expert participants, who had a long history and substantive knowledge of the Indigenous ELCC landscape in BC. To increase the policy relevance and impact of the findings and recommendations at a provincial government level, a draft summary of the research was also shared with the Indigenous Engagement and Policy Branch of the Provincial Office for the Early Years.

\section{Limitations}

The recruitment strategy resulted in certain regions of BC being represented more than others. As outlined above, to overcome this limitation, two Indigenous ELCC experts with a long history of working in the Indigenous ELCC 
sector in $\mathrm{BC}$ were recruited to provide provincial overviews and insights related to this project. The scope and funding of this study did not allow for examining issues related to operational successes or challenges from the perspectives of Indigenous families or children.

\section{Findings}

The findings highlight some of the key strengths and challenges of operating Indigenous ELCC programs. In the following section, the main themes and related subthemes are discussed and linkages made to the literature. These include (1) program capacity to "set up a strong foundation"; (2) programming "takes a lot of money"; and (3) staffing is "our number one operational challenge."

\section{Program capacity to "set up a solid foundation"}

Research participants described a successful and high-quality Indigenous ELCC program as one that "sets up a solid foundation [and] requires ... investment of time, resources, funding, energy and attention to caring for the caregiver" (P11). Identified interrelated subthemes highlighting key characteristics of quality programs that "set up a solid foundation" are summarized in Figure 1 and discussed thereafter.

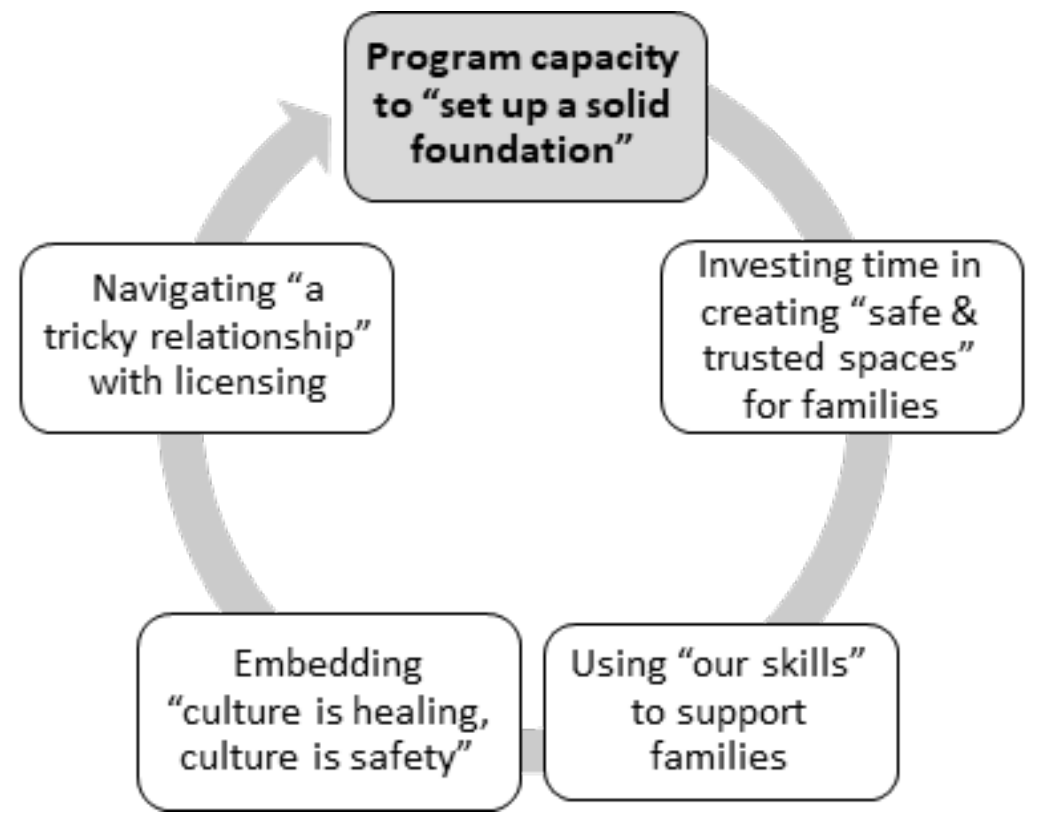

Figure 1. Summary of subthemes in "program capacity to set up a solid foundation."

Investing time in creating "safe and trusted spaces" for families. Consistent with the spirit of Friendship Centres, participants talked about the importance of focusing their time, efforts, and resources on providing "safe and trusted spaces" for families and children. As an ED commented:

We had the evaluators come in to evaluate the new universal childcare programs. And all the families met, and there was no staff in the room, and the thing that they said was the best part of the program was that they felt safe there and they felt their children were safe there. (P6)

The capacity to "create safe and trusted spaces" was in large part dependent on programs being able to recruit staff who were the "right fit" and retain them over the long term in order to provide continuity in family-staff 
relationships:

We want those caregivers to be consistent, to be long term, because it's based on relationship. The families look to these educators as aunties, uncles, as surrogate aunties and uncles, extensions of their family. But if these people keep leaving and new staff keep coming on you're not creating that familiar place, the continuity. And really more replicating the areas that challenge families of inconsistent people in their lives, lacking continuity, but if we can demonstrate in our delivery of early years programs continuity and knowing we're reliable, we also are then creating those trusting spaces and demonstrating ... healthy relationships and healthy expectations. (P11)

This theme highlights the critical link between programs being able to retain qualified staff and their capacity to foster a sense of safety and trust with families. Staff retention is an important concern for many ELCC contexts (Totenhagen et al., 2016) but is perhaps even more salient in Indigenous contexts due to parental concerns about and distrust towards institutional care for their children (Hare \& Anderson, 2010).

Using "our skills" to support families. "Setting up a solid foundation" also required that staff responded to families' priorities and needs beyond child care. As one participant commented, "I tell everyone that we don't run childcare centres, we run family centres." (P16) Another added:

It's just stepping out of our roles as daycare providers and looking at how we use our skill sets to support families.... What we need to do is we need to address the family's concerns first, support them in their concerns and then over time, once they have those things dealt with, they're able to open up to the other things that we may offer. (P18)

Participants described needing the time and resources to respond to multifaceted social challenges faced by families and children in their programs, including "children who are either in care or in jeopardy of being in care" (P1), "a lot of poverty" (P18) and housing insecurity, intergenerational family histories of child removal, and young children who have experienced trauma and/or have "special needs."

This data points to the importance of programs and staff investing time and resources in responding to each family's circumstances and priorities. This is consistent with a Canada-wide 2017 study with AHSUNC which concluded that programs strengthen Indigenous community resilience by emphasizing healthy child development in the context of a child's social system, focusing not only on the child but on their family and the larger community (Public Health Agency of Canada, 2017). However, this finding also raises concerns that many programs do not have the financial resources to invest in a family support worker position to provide this essential form of support.

Embedding "culture is healing, culture is safety." Not surprisingly given the existing evidence discussed in the introduction, "setting up a solid foundation" was also dependent on the capacity of programs to foster families and children's long-term well-being by "reconnecting and reclaiming cultural identity and language" (P10), providing opportunities for families to "express themselves in all the myriad of cultural ways" (P12), and "families being proud of being Indigenous and wanting to ... live that life as best they can" (P12). Elder involvement was viewed as "a critical piece to everything we do" (P12). An ED reflected:

Culture is healing, culture is safety. Connection is safety. The more positive connections we build around these children earlier in life the less likely they'll end up in a system that's probably going to eat them up and spit them out. (P10)

While the fundamental right of Indigenous children to have access to Indigenous ELCC programming is well established, including by the Royal Commission on Aboriginal Peoples (1996), UNDRIP (2007), and the TRC 
(2015), the actual implementation of this right requires funding. As a program manager stated:

With everything that we work so hard to do for our families the one thing I'm really proud of is that we are really answering the TRC's Call to Action for the families. Like we are doing it. We have everything in place and what l'd really love to get the message back to the government is-if you're serious about the Call to Action then you really need to be making sure that our demands for what we're looking for, for our centres, for our families are being answered. (P12)

Participants' perspectives on having the capacity and financial resources to employ Elders and knowledgeable community members and to anchor their programming in local Indigenous cultures and languages are consistent with and support previous research with AHSUNC programs in Canada (Public Health Agency of Canada, 2017). Moreover, capacity development is a key principle and required action to meet the legislated obligations of UNDRIP (United Nations, 2007) in BC and the national Indigenous ELCC Framework (Government of Canada et al., 2018). In this subtheme, licensing was also raised as a related concern.

Navigating “a tricky relationship” with licensing. In BC, the Community Care and Assisted Living Act (Queen's Printer, 2002), the Residential Care Regulation (Queen's Printer, 2009), and the Child Care Licensing Regulation (Queen's Printer, 2007) establish the minimum health and safety requirements that must be met. Licensed childcare programs are monitored and regularly inspected by regional health authority licensing officers to ensure programs are meeting specific requirements for health and safety, staffing qualifications, record keeping, space and equipment, child-to-staff ratios, and programming (Government of British Columbia, 2008). The purpose of licensing is to ensure "the provision of quality child care services in British Columbia ... [and] protect the health and safety of children in care" (Government of British Columbia, 2008). Provincial funding is contingent on programs being licensed.

In this study, several participants described how they had to navigate a potentially "tricky relationship" with their licensing officer, with one saying, "I think that it's a tricky relationship for sure. No matter what I think, even if you have a really good relationship with them, there's always going to be something else and that's their job" (P9). Participants expressed concerns that the licensing approval process was dependent on a licensing officer's personal interpretation of the legislation and the strength of their relationship. Experiences reflected a variation in their relationships with licensing officers:

Unfortunately, what I am seeing is that some of that is dependent on the licensing officer perspective and attitude and it shouldn't be, right? It should be a very consistent regulation across the board, but we're finding that it's not. (P16)

We're really fortunate, we've had the same licensing officer since we opened, so we have an amazing relationship with her and ... she trusts how we work and operate and knows that we're a stand-up program. So, an example would be other places aren't allowed to use traditional food because it's not licensed approved, but if we get parents to sign off on a letter saying that they're okay with their child eating traditional food, we're allowed to serve it. (P4)

The implementation of government-funded ELCC programs involves complying with an array of predetermined structural requirements that may constrain or support the inclusion of Indigenous-specific attributes in the face of no Indigenous-specific authority (Greenwood et al., 2007). Licensing is one example of a value- and culturally laden structural requirement that does not distinguish Indigenous ELCC programs from other ELCC programs. Participants' concerns about licensing echo those raised by the BC Aboriginal Child Care Society (2013) and the Indigenous ELCC Framework (Government of Canada et al., 2018). For example, a key indicator of quality in Indigenous ELCC programs is Elder involvement. As knowledge holders, Elders are indispensable in cultural 
programming, but their value is not always recognized in licensing regulations (BC Aboriginal Child Care Society, 2014b).

\section{Programs "take a lot of money"}

An affirming and not surprising main theme related to the operational challenge of urban Indigenous ELCC programs was that programs "take a lot of money." As the following participants note, investing in an ELCC program is a long-term venture, and the "returns" on this investment may not be fully evident for several years:

They [programs] do take a lot of money to run, as you can imagine, especially being a no fee service. But the outcomes and the cost reduction on the other side of the prevention plan is showing that spending the money upfront is working. (P12)

There needs to be an understanding that childcare requires a lot of resources to be high quality. It is not a financial gaining endeavour. It will deplete resources more than contribute. [But] the resources it contributes are higher-better opportunities for children and families with high-quality experiences. Then understanding that, you aren't going to make money delivering childcare, but the outcome of investing in childcare is huge.... The advice to Friendship Centres is, if you don't have a childcare program and you want one, you need to be well positioned before opening one and going into that knowing and recognizing that it's going to use up services and added resources and funding rather than contribute to the pot. (P11)

Within this theme, two interrelated subthemes were identified, as summarized in Figure 2.

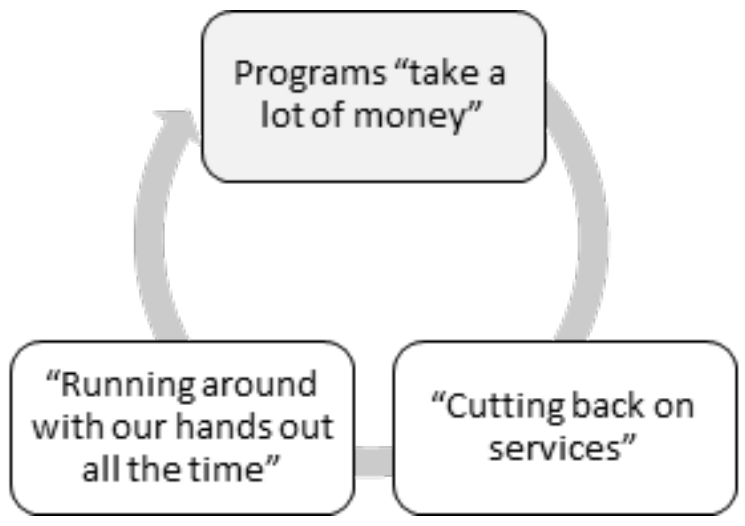

Figure 2. Summary of subthemes in programs "take a lot of money."

Having to "cut back on services." The historical stagnation in funding for urban Indigenous ELCC programs in BC has resulted in programs "cutting back on services" in order to stay open and/or within budget. As the following participant described:

Well, we try to cut down some staff hours, so like our family involvement workers are part time now, our bus driver is part time, our cook is part time, and those all used to be full-time positions ... putting money back into a facility and making sure that it's taken care of, but it's also not just that-it's like how do you do technological upgrades, you know, computers, telephones, cell phones, you know, all of that other infrastructure that stuff ages and you have to be able to replace it. (P4)

Also, when programs are dependent on parent fees, the financial and administrative burden on programs and host organizations increases, and providing a quality program can be compromised: 
The program is very challenged by [a] limited budget. Because it operates on parent fees, the income for their budget is low and cannot address the ongoing needs for the childcare [program] to deliver enhanced program and curriculum. The staff reported that they have not received any supply budgets and therefore have to do without or purchase materials with their own money. Also, there is no funds to have janitorial support so the staff focus a lot of time in general daily maintenance of the building missing out on key learning opportunities with the children. (P11)

This finding is consistent with the recommendations in the Indigenous ELCC Framework (Government of Canada et al., 2018) calling for appropriate and stable funding that reflects the realities, fundamental rights and responsibilities of operating quality Indigenous ELCC programs for a growing urban population. As previously discussed, historically rooted jurisdictional ambiguities and wrangling over which level of government (federal or provincial) is responsible for funding urban Indigenous ELCC has resulted in the stagnation of funding for decades (BC Aboriginal Child Care Society, 2014a). This lack of government investment fails to address a fastgrowing urban Indigenous population whose children, as previously discussed, have the legal right to ELCC that is grounded in local Indigenous and community cultures, languages, protocols, practices, and histories.

"Running around with our hands out all the time." Primary funding sources for the programs in this study included parents' fees, the provincial government, PHAC, and individual grant applications crafted by host organizations. The current capacity of many programs to meet their operating costs was dependent on executive directors spending large amounts of time writing proposals, competing for limited funds, and reporting back to multiple funders in order to cover the operational costs of their ELCC programs—creating what the First Nations ELCC Policy Framework refers to as an "administrative burden" (BC Aboriginal Child Care Society, 2017). As the following executive director stated:

We're operating in the red all the time. So, its taxing to the organization as a whole to keep it open... We run around with our hands out all the time and we apply for every possible [funding name] that we possibly can which takes a huge amount of staff time. It's difficult when you put say 28 hours in a proposal and you get nothing back in return. That happens way too often. (P15)

This finding also affirms that building or maintaining programs' capacity, particularly in communities that do not have the resources to write strong proposals, requires sustainable, long-term, and noncompetitive funding to avoid perpetuating inequities between communities - that is, underresourced communities get less and betterresourced communities get more. As this executive director notes:

We need more sustainable funding. But one of the problems l've faced in my 20 years of service in communities was the competitive nature of funding. The system is set up that communities have to compete against each other to get pots of money. Whoever writes the best proposal, whoever has the best infrastructure is able to access the money. (P18)

The current inadequate funding available for operating costs results in many executive directors having to compete and be accountable to multiple funders in order to keep their program running, which requires significant time and resources with no guarantee that funding will be forthcoming or stable. This finding raises questions about a single-source funding model as an administrative and operational matter whilst also upholding the rights and mandate of Indigenous peoples to determine how funding is allocated rather than government authorities (Government of Canada et al., 2018).

Staffing is "our number one operational challenge"

There was some variation in the data on programs' reported capacities to keep their staff. Some participants 
reported having staff in their programs for 10 years or more, and other participants described not being able to keep staff beyond two years. These different experiences may relate to the data discussed in a later section, on the ability of programs to support staff's ongoing professional development and well-being and a living wage. Regional variations may also account for different experiences in recruiting and retaining staff. For example, in the lower mainland of BC, ECEs are more mobile and have multiple employment options. Recruiting and keeping ECE staff in this region and job market may therefore be more challenging. Nonetheless, a recurring theme in the data identified that "our number one challenge operationally is staffing" (P16). Or, as the following participant pointed out, "the biggest issue is qualified staff. Previous governments have said we're going to invest money and build new spaces. You can build the building, you can fill it full of children, but you cannot staff it" (P18). Within this broad theme, analysis of the data identified three interrelated subthemes, summarized in Figure 3 and discussed in the following section.

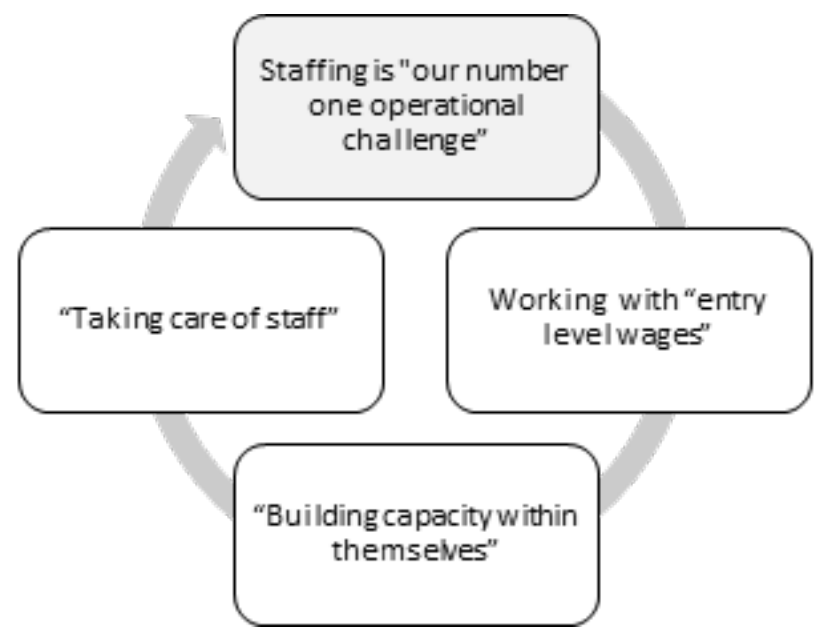

Figure 3. Summary of subthemes in staffing is "our number one operational challenge."

Working with “entry-level wages.” Participants frequently linked challenges with staff recruitment and retention with the burden of low wages for ECEs. As the following participant summed up, "the average person that works in the daycare only sticks around for two years. It's an entry level job. .. and they don't stick around very long because the wages aren't sufficient" (P1003). Other participants noted:

The wage people are making [is] the same wage as a check-out clerk. And so why should they go to college, why should they take on the extra burden and stress of being an early child educator when they [can] simply go and be a check-out clerk and make the same money and have better benefits? And it's always been a challenge and we are hitting a wall. There is not enough educators out there. Many programs are struggling just to staff their programs with regular employees and many places have closed because they cannot staff it or find substitutes. (P18)

What we have been told is that a lot of people have not gotten their infant and toddler [certification] because it really wasn't much of an increase in wage for them. So, it didn't feel like it was worth their while to spend another year or two years to get the certificate. And so, yeah, we're finding that that is a challenging position to get. We have one now. We had two before and we lost both of them. (P1)

As previously mentioned, numerous initiatives are underway to incentivize ECEs to maintain their employment in $\mathrm{BC}$, including a provincial wage enhancement initiative that equates to an extra $\$ 2$ an hour (Government of British Columbia, 2018b), a higher hourly rate through the "growing Aboriginal Head Start" federal initiative (Aboriginal 
Head Start Association of BC, 2019), and wage enhancement as part of an Indigenous ELCC investment strategy (BC Aboriginal Child Care Society, 2019). It is too early to know how effective such strategies will be in recruiting and retaining Indigenous staff in Indigenous ELCC programs.

"Building capacity within themselves." Participants discussed how they mitigated the operational challenges related to recruiting and/or keeping staff and providing quality programming by "building staff's capacity within themselves" - in other words, supporting and investing in staff's ongoing professional development. The following ED identified finding the funding for staff education as a high priority and ongoing challenge that often depends on the success of a funding application:

My job is to ensure that we have the best trained staff that we can and ... to build capacity in them because I want a high-quality program [because] our children deserve the best care that we can give them. And so, always working with the staff to try and complete their ECE.... We could use ten more ECEs definitely, but our staff is really seeing the need to build capacity within themselves and to help out the needs of the centre. So, we have five staff right now working on their infant/toddler and we're supporting that. We're helping them pay for their courses, helping them pay to do their practicums, trying to keep it in house as much as possible. So, it feels really good to know that staff are that committed to the program.... When I build the budget I definitely push the need to our funders that we need training dollars and those need to be there to help us with that. And the funders are really good about that, you know, here's why we need these dollars, it's important. We're building capacity, we're doing better work for our families when we have better trained staff, and the funders are really good about that. (P7)

This participant went on to highlight the need for more work-integrated learning opportunities to support professional development while also attending to the need for continuity, as previously discussed:

It hurts me to see people having to take six weeks off work to do a practicum in our province. We need people working and we need to find a way that they can train where they're working, because why take them away for six weeks and pull them out and the children lose that person? Our kids can't lose people, so we need that continuity and consistency for those children and those families, but we also need to figure out a way that we can train staff in their workplace. (P7)

The subtheme of "building capacity within themselves" also included participants' perspectives on the need for more opportunities for ELCC programs to "build capacity within themselves" by have stronger relationships with similar programs in their region and different parts of the province.

This finding shows that, although it is well established that the ongoing professional development of ELCC staff is a significant predictor of program quality (Government of Canada, 2017), accessing ongoing professional development continues to be a challenge for many urban Indigenous ELCC programs and it will therefore be important to monitor the impact of the provincial government's recently announced innovations. A promising and recently formed First Nations Pedagogies Network in BC (https://fnpn.ca/) is an example of an Indigenous-led initiative to support to support ECEs in their ongoing professional development in the context of urban Indigenous ELCC programs. Staff retention is also closely aligned with staff well-being, which is explored in the following subtheme.

“Taking care of staff." Participants talked about the importance of taking care of staff in order to keep long-term staff and provide a quality program. As one participant clearly stated, "your staff need to be taken care of so that that they can give quality childcare" (P5). One manager highlighted a proactive team approach: 
So the staff are generally happy with the job satisfaction, our job hours, and the pay. We're able to have benefits as well. And I think our new management team in place is really proactive. We have a really good team setting now. So, going forward I think we're going to see really good retention in our staff and that we've really worked hard on that the last couple of years to make sure that our staff were happy. (P12)

Staff satisfaction also related to relationships and communication in programs so that staff had "a voice" to inform and be involved in change. As one ED noted:

Making sure that you're communicating with your staff regularly. It's really hard to make time to do that in a daycare situation because they're so busy ... but you don't know what's going on in here (signalling with hands the space in a classroom) unless you're having conversations. And if you don't give opportunities to have those conversations, whatever is going on just builds up and you don't want that.... We've implemented regular staff meetings and that's another thing that we think is very, very important for our staff to have a voice. But we also want to make sure that their voice is about change it's not about just complaining about situations but it's about also coming forward with some recommendations about how they see that change happening. And for us to act on that as quickly as possible but also have them involved then in the change. (P1)

The need for funding for staff wellness as a strategy for keeping staff longer was also identified:

If I could do more wellness as a preventative measure it would make a big difference because right now they [program staff] don't like taking time off because we don't have enough subs and also because we don't have that on our contract to offer them. But wellness is so important when you're working with our little guys, right? You have to come with 150\%, you can't come with less than that. And when you have less then they know it. And when you have less then your team also is affected. And so the more people I have coming with less, the whole team is impacted, their stress gets higher. Like if I had more wellness happening then l'd definitely have a healthier staff that wanted to stay longer. (P8)

In the context of operating quality Indigenous ELCC programs described in this study, including the critical importance of retaining long-term staff, "taking care of staff" is a priority that can get overlooked given the multiple demands on everyone's time and energy and the financial constraints. "Taking care of staff" from a strengths-based perspective can also encompass having programs that foster job role clarification, appreciation of strengths and work completed, a sense of teamwork and team cohesion, and good communication between staff and management (Deroy \& Schütze, 2019). Finally, the findings in "staffing is our number one operational challenge" point to the urgent need for the BC government to enact their stated commitment to UNDRIP and redress the health and social inequities experienced by many young Indigenous children by advancing Indigenous governance of urban ELCC programs with stable and appropriate funding for staffing and operations.

\section{Discussion}

A robust system of quality Indigenous ELCC can make a vital contribution towards redressing the legacies of colonialism across Indigenous children's life course (BC First Nations Early Childhood Development Council, 2009; Government of Canada et al., 2018; Greenwood \& de Leeuw, 2012; Public Health Agency of Canada, 2012, 2016). Quality Indigenous ELCC programs are controlled, planned, designed, and delivered by Indigenous organizations and communities (Government of Canada et al., 2018; Halseth \& Greenwood, 2019). However, consistent with existing evidence (BC Aboriginal Child Care Society, 2014a; Government of Canada et al., 2018), the operational challenges highlighted in this paper show that the potential of urban Indigenous ELCC to make a substantial difference in the life chances of Indigenous children continues to be denied in the current system of 
state control and inadequate funding.

To achieve social and health equity for Indigenous children, political action and sustainable funding is required to support a transition to "the design, delivery and governance of Indigenous ELCC that is anchored in selfdetermination" (Government of Canada et al., 2018, p. 5). This transition of authority enacts Indigenous peoples' legislated right to self-determination and Indigenous children's right to culturally relevant curricula and heritage languages in accordance with the TRC Calls to Action (Truth and Reconciliation Commission of Canada, 2015), the UNCRC (United Nations, 1989), and UNDRIP (United Nations, 2007). The transition to self-determination and governance of Indigenous ELCC is particularly salient in the BC context, given that BC, in 2019, was the first jurisdiction in Canada to pass legislation to implement UNDRIP.

The operational challenges of staff retention outlined in this paper expand on current global discourses on this topic (Pek-Greer \& Wallace, 2017; Totenhagen et al., 2016) by highlighting the essential continuities in Indigenous ELCC programs between staff retention and the capacity of programs to create "safe and trusting spaces" for families and children. This is of critical importance given that Canada's history of forced child removal, which disrupted generations of Indigenous families, continues to generate widespread mistrust about engaging with formalized child-related programs (Gerlach, Browne, \& Greenwood, 2017; Hare \& Anderson, 2010). The potential positive impacts of engaging Indigenous families and children in Indigenous ELCC programs are therefore contingent on programs having sustainable funding in order to retain staff and foster long-term community-family-staff relationships. This finding provides further evidence for an adequate and stable one-source funding model that provides a living wage for staff and allows for professional development and strategies to support staff wellness. When ELCC programs have adequate and secure funding, qualified staff are likely to retain long-term positions that provide "a solid foundation for families" grounded in sustained, trusting relationships.

Consistent with the Friendship Centre ethos, the findings in this paper also highlight that the operating costs of urban Indigenous ELCC programs need to respond to the socioeconomic realities and priorities of urban Indigenous families' lives. As this study shows, providing "a strong foundation" for Indigenous families and children involves a broader scope of ELCC that requires staff to "use their skill sets" to support the needs of the family as a whole. Consistent with Indigenous philosophies on raising healthy children (Halseth \& Greenwood, 2019) and the capacity of Indigenous ELCC programs to promote family well-being (Gerlach, Browne, \& Suto, 2018; Gerlach \& Gignac, 2019)2018; Gerlach \&amp; Gignac, 2019, the findings reinforce the need for operational costs to include funding for designated family support workers. Investing in designated family support workers strengthens the capacity of programs to mitigate the impacts of poverty on children's early health, development, and life experiences.

\section{Conclusion}

Quality Indigenous designed and delivered ELCC programs play a vital role in achieving social and health equity for Indigenous children, their families and communities (Halseth \& Greenwood, 2019). However, as the findings in this paper show, the longstanding operational challenges of urban, not-for-profit Indigenous ELCC programs in $\mathrm{BC}$ are kept in place by a failure of all levels of government to implement their legislated obligations to UNDRIP (United Nations, 2007), the UNCRC (United Nations, 1989), and the Calls to Action of the TRC (Truth and Reconciliation Commission of Canada, 2015). Enacting these obligations requires that provincial and federal governments make greater investment in Indigenous ELCC programs and the growing number of Indigenous families that are choosing to raise their children in urban centres. There also needs to be strategic action and investment in "research and evaluation frameworks to support promising practice and innovations in Indigenous 
ELCC policies, programs, and services" (Government of Canada et al., 2018, p. 13). Ultimately, as outlined in the Indigenous ELCC Framework (Government of Canada et al., 2018), strategic transformations in ELCC structures, operations, and programs that are controlled and directed by Indigenous leadership are fundamental to redressing the intergenerational impacts of colonialism and realizing the potential of Indigenous ELCC programs to make a difference in the life chances of Indigenous children in this country.

\section{Acknowledgements}

This research was funded by the Social Sciences and Humanities Research Council (SSHRC) as part of the Western Centre of the Urban Indigenous Research Network. The authors would like to sincerely thank all of the participants who were so generous in sharing their time, knowledge, and passion for their early learning and child care programs.

${ }^{1}$ The term Indigenous is used in this paper to refer inclusively to all First Nations, Inuit, and Métis peoples who have their own diverse identities, histories, rights, and relationships to the Canadian state. 


\section{References}

Aboriginal Head Start Association of BC. (2019). Growing AHS in BC. https://www.ahsabc.com/growinginbc

BC Aboriginal Child Care Society. (2013). Licensing First Nations' early childhood programs. Research report prepared for the First Nations Early Childhood Development Council. https://www.acc-society.bc.ca/wp-content/uploads/2018/06/bcaccs-licensingreport-FINAL-October-15-2013.pdf

BC Aboriginal Child Care Society. (2014a). An environmental scan of public policy and programs for young Aboriginal children in BC: A cold wind blows. https://www.acc-society.bc.ca/files_2/documents/BCACCSENglobalscanFINAL.pdf

BC Aboriginal Child Care Society. (2014b). What you need to know about the inclusion of Elders in early childhood development settings. http://www.acc-society.bc.ca/files 2/documents/InclusionofEldersInfoFINALCOPY reduce.pdf

BC Aboriginal Child Care Society. (2017). National First Nations early learning and child care policy framework. https://gallery.mailchimp. com/a74eaafbca72d669859e6453b/files/2abc3f2b-822e-4cb6-860b-cf282e7f8975/First Nations ELCC draft Framework November 17.pdf

BC Aboriginal Child Care Society. (2019). Indigenous early learning and child care investment in BC: An overview. http://www.acc-society. bc.ca/wp-content/uploads/2019/12/Investment-Strategy-Overview-to-be-printed-as-PDF-updated-Dec11.2019.potx.pdf

BC First Nations Early Childhood Development Council. (2009). BC First Nations early childhood development framework. http://www. nscr.bc.ca/childcare/resource-pdf/First\%20Nations\%20ECD\%20Framework.pdf

Bell, R., Donkin, A., \& Marmot, M. (2013). Tackling structural and social issues to reduce inequities in children's outcomes in low-to middleincome countries. Office of Research Discussion Paper No.2013-02. https://www.unicef-irc.org/publications/pdf/marmot layout5.pdf

Boyce, W. T., \& Hertzman, C. (2018). Early childhood health and the life course: The state of the science and proposed research priorities. In N. Halfon, C. B. Forrest, R. M. Lerner, \& E. M. Faustman (Eds.), Handbook of life course health development (pp. 47-93). Springer https://doi.org/10.1007/978-3-319-47143-3

Britto, P. R., Yoshikawa, H., \& Boller, K. (2011). Quality of early childhood development programs in global contexts: Rationale for investment, conceptual framework, and implications for equity. http://srcd.org/sites/default/files/documents/spr v252rev.pdf

CTV News. (2018, June 5). Parents raise questions after daycare’s decision to close (updated July 10). https://bc.ctvnews.ca/parents-raisequestions-after-daycare-s-decision-to-close-1.3961075

Deroy, S., \& Schütze, H. (2019). Factors supporting retention of Aboriginal health and wellbeing staff in Aboriginal health services: A comprehensive review of the literature. International Journal for Equity in Health, 18(70). https://doi.org/10.1186/s12939-019$\underline{0968-4}$

First Nations Information Governance Centre. (2012). First Nations regional health survey (RHS): Phase 2 (2008/10) National report for adults, youth and children living in First Nations communities. http://fnigc.ca/sites/default/files/docs/first nations regional health survey rhs 2008-10 - national report.pdf

Gerlach, A. J. (2007). Steps in the right direction: Connecting and collaboration in early intervention therapy with Aboriginal families and communities in British Columbia. http://www.acc-society.bc.ca/files new/documents/ StepsintheRightDirectionConnectingandCollaboratinginEarlyInterventionTherapywithAb.Familiesa.pdf

Gerlach, A. J., Browne, A. J., \& Greenwood, M. (2017). Engaging Indigenous families in a community-based early childhood program in British Columbia, Canada: A cultural safety perspective. Health \& Social Care in the Community, 25(6), 1763-1773. https://doi. org/10.1111/hsc. 12450

Gerlach, A. J., Browne, A. J., \& Suto, M. J. (2018). Relational approaches to fostering health equity for Indigenous children through early childhood intervention. Health Sociology Review, 27(1), 104-119. https://doi.org/10.1080/14461242.2016.1231582

Gerlach, A. J., \& Gignac, J. (2019). Continuities between family engagement and wellbeing in Aboriginal Head Start Programs in Canada: 
A qualitative inquiry. Infants and Young Children, 32(1), 60-74. https://doi.org/10.1097/IYC.0000000000000133

Gerlach, A. J., Gulamhusein, S., Varley, L., \& Perron, M. (2019). Setting up a solid foundation: Exploring the capacity of Indigenous notfor-profit early learning and child care programs in British Columbia-A summary report. https://bcaafc.com/wp-content/ uploads/2020/01/Setting-Up-a-Solid-Foundation-Nov2019.pdf

Government of British Columbia. (2008). Child care licensing regulation: Information package. https://www.health.gov.bc.ca/library/ publications/year/2008/Child Care Licensing_Regulation.pdf

Government of British Columbia. (2018a). Child care BC caring for kids, lifting up families: The path to universal child care plan. https:// www.bcbudget.gov.bc.ca/2018/childcare/2018 Child Care BC.pdf

Government of British Columbia. (2018b). Investing in our early childhood educators: Early care and learning recruitment and retention strategy. https://www2.gov.bc.ca/assets/gov/family-and-social-supports/child-care/6337 earlycareandlearningrecruitment andretentionstrategy_report_web.pdf

Government of British Columbia. (n.d.). Urban and off-reserve Aboriginal people. https://www2.gov.bc.ca/gov/content/governments/ indigenous-people/supporting-communities/urban-off-reserve-aboriginal-people

Government of Canada. (2017). Multilateral early learning and child care framework https://www.canada.ca/en/employment-socialdevelopment/programs/early-learning-child-care/reports/2017-multilateral-framework.html

Government of Canada, Assembly of First Nations, Inuit Tapiriit Kanatami, \& Métis National Council. (2018). Indigenous early learning and child care framework. https://www.canada.ca/en/employment-social-development/programs/indigenous-earlylearning/2018-framework.html

Greenwood, M. (2005). Children as citizens of First Nations: Linking Indigenous health to early childhood development. Pediatric Child Health, 10(9), 553-555.

Greenwood, M. (2016). Language, culture, and early childhood: Indigenous children's rights in a time of transformation Canadian Journal of Children's Rights, 3(1). http://journals.carleton.ca/cjcr/index.php/cjcr/article/view/95/147

Greenwood, M., \& de Leeuw, S. (2007). Teachings from the land: Indigenous people, our health, our land, and our children. Canadian Journal of Native Education, 30(1), 48-53, 189.

Greenwood, M., \& de Leeuw, S. (2012). Social determinants of health and the future wellbeing of Aboriginal children in Canada. Paediatric Child Health, 17(7), 381-384. https://www.ncbi.nlm.nih.gov/pmc/articles/PMC3448539/

Greenwood, M., de Leeuw, S., \& Fraser, T. (2007). Aboriginal children and early childhood development and education in Canada: Linking the past and the present to the future. Canadian Journal of Native Education: Special Issue on Early Childhood, 30(1), 5-18.

Greenwood, M., \& Jones, E. (2015). Being at the interface: Early childhood as a determinant of health. In M. Greenwood, S. de Leeuw, N. M. Lindsay, \& C. L. Reading (Eds.), Determinants of Indigenous peoples' health in Canada: Beyond the social (pp. 64-77). Canadian Scholars' Press.

Hacker, K. (2013). Community-based participatory research. SAGE.

Hajizadeh, M., Hu, M., Bombay, A., \& Asada, Y. (2018). Socioeconomic inequalities in health among Indigenous peoples living offreserve in Canada: Trends and determinants. Health Policy, 122, 854-865. https://doi.org/10.1016/j.healthpol.2018.06.011

Halfon, N., Forrest, C. B., Lerner, R. M., \& Faustman, E. M. (Eds.). (2018). Handbook of life course health development. Springer. https:// doi.org/10.1007/978-3-319-47143-3.

Halseth, R., \& Greenwood, M. (2019). Indigenous early childhood development in Canada: Current state of knowledge and future directions. National Collaborating Centre for Aboriginal Health. https://www.nccah-ccnsa.ca/docs/health/RPT-ECD-PHAC-GreenwoodHalseth-EN.pdf 
Hare, J., \& Anderson, J. (2010). Transitions to early childhood education and care for indigenous children and families in Canada: Historical and social realities. Australasian Journal of Early Childhood, 35(2), 19-27.

Lake, A., \& Chan, M. (2014). Putting science into practice for early child development. The Lancet, 385(9980), 1816-1817. https://doi. org/10.1016/S0140-6736(14)61680-9

Newhouse, D., \& Peters, E. (2003). Not strangers in these parts: Urban Aboriginal peoples. Policy Research Initiative. http://www. urbancentre.utoronto.ca/pdfs/elibrary/Canada_Urban-Aboriginal-Peo.pdf

Pek-Greer, P., \& Wallace, M. (2017). A study of childcare teacher retention in the childcare service industry. Global Business Review, 18(1), 71-86. https://doi.org/10.1177\%2F0972150916666879

Place, J. (2012). The health of Aboriginal people residing in urban areas. National Collaborating Centre for Aboriginal Health. http://www. nccah-ccnsa.ca/Publications/Lists/Publications/Attachments/53/Urban Aboriginal Health EN web.pdf

Public Health Agency of Canada. (2012). The impact of the Aboriginal Head Start in Urban and Northern Communities (AHSUNC) Program on school readiness skills. http://www.mbaboriginalheadstart.ca/ mndata/mbheadstart/uploaded files/AHSUNC\%20 School\%20Readiness\%20Study.pdf

Public Health Agency of Canada. (2016). Aboriginal Head Start in Urban and Northern Communities: Closing the gap in health and education outcomes for Indigenous children in Canada. https://www.canada.ca/content/dam/hc-sc/documents/services/ publications/healthy-living/aboriginal-head-start/closing-the-gap-fact-sheet-en.pdf

Public Health Agency of Canada. (2017). Aboriginal Head Start in Urban and Northern Communities: Further strengthening Indigenous community resilience. http://publications.gc.ca/collections/collection 2018/aspc-phac/HP35-82-2017-eng.pdf

Reading, C. L., \& Wien, F. (2013). Health inequalities and social determinants of Aboriginal peoples' health. National Collaborating Centre for Aboriginal Health. https://www.ccnsa-nccah.ca/docs/determinants/RPT-HealthInequalities-Reading-Wien-EN.pdf

Royal Commission on Aboriginal Peoples. (1996). Report of the Royal Commission on Aboriginal peoples, Volume 3: Gathering strength. https://www.bac-lac.gc.ca/eng/discover/aboriginal-heritage/royal-commission-aboriginal-peoples/Pages/final-report.aspx

Shonkoff, J. P. (2012). Leveraging the biology of adversity to address the roots of disparities in health and development. Proceedings of the National Academy of Sciences, 109(2), 17302-17307. https://doi.org/10.1073/pnas.1121259109

Smylie, J., \& Adomako, P. (2009). Indigenous children's health report: Health assessment in action. Centre for Research on Inner City Health. http://www.stmichaelshospital.com/crich/indigenous childrens health report.php

Statistics Canada. (2017). Aboriginal peoples in Canada: Key results from the 2016 census. http://www.statcan.gc.ca/daily-quotidien/171025/ dq171025a-eng.pdf

Stout, M. D. (2012). Ascribed health and wellness, Atikowisi miyw-ayawin, to achieved health and wellness, Kaskitamasowin miywayawin: Shifting the paradigm. Canadian Journal of Nursing Research, 44(2), 11-14. http://www.ingentaconnect.com/content/ mcgill/cjnr/2012/00000044/00000002/art00003

Tait, C. L., Henry, R., \& Loewen Walker, R. (2013). Child welfare: A social determinant of health for Canadian First Nations and Métis children. Pimatisiwin: A Journal for Aboriginal and Indigenous Community Health, 11(1), 39-53. https://www.academia. edu/11323340/Child_Welfare_A_Social_Determinant_of_Health_For_Canadian_First_Nations_and_M\%C3\%A9tis_ Children

Totenhagen, C. J., Hawkins, S., Casper, D. M., Bosch, L. A., Hawkey, K. R., \& Borden, L. M. (2016). Retaining early childhood education workers: A review of the empirical literature. Journal of Research in Childhood Education, 30(4), 585-599. https://doi.org/10.10 $\underline{80 / 02568543.2016 .1214652}$

Truth and Reconciliation Commission of Canada. (2015). Honouring the truth, reconciling for the future: Summary of the final report of the Truth and Reconciliation Commission of Canada. http://nctr.ca/assets/reports/Final\%20Reports/Executive Summary English Web.pdf 
UNICEF. (2014). Brain development in children: The impact of adversity. UNICEF Connect. http://blogs.unicef.org/2014/04/15/braindevelopment-in-children-the-impact-of-adversity/

United Nations. (1989). Convention on the rights of the child. https://www.ohchr.org/en/professionalinterest/pages/crc.aspx

United Nations. (2007). UN declaration on the rights of Indigenous peoples. http://www.un.org/esa/socdev/unpfii/documents/DRIPS en.pdf

Wagner, S. L., Forer, B., Cepeda, I. L., Goelman, H., Maggi, S., D’Angiulli, A., Wessel, J., Hertzman, C., \& Grunau, R. E. (2013). Perceived stress and Canadian early childhood educators. Child \& Youth Care Forum, 42, 53-70. https://psycnet.apa.org/doi/10.1007/ $\underline{\text { s10566-012-9187-5 }}$

Wilk, P., Maltby, A., \& Cooke, M. (2017). Residential schools and the effects on Indigenous health and well-being in Canada: A scoping review. Public Health Reviews, 38(8), 1-23. https://doi.org/10.1186/s40985-017-0055-6 